\title{
Análise de Eficiência das Empresas Agrícolas do Pólo Petrolina/Juazeiro Utilizando a Fronteira Paramétrica Translog
}

Emanoel de Souza Barros*

Ecio de Farias Costa**

Yony Sampaio***

Resumo: A irrigação iniciada no final da década de sessenta foi a principal responsável pelo marcante desenvolvimento ocorrido no pólo Petrolina/Juazeiro. Com a irrigação chegaram empresas agrícolas para a região, antes voltadas para outras atividades, como a pecuária extensiva. Supõe-se, então, que a moderna tecnologia usada na irrigação, combinada com a utilização de insumos, permite um nível de produção próximo ou sobre a fronteira de produção. Estudos preliminares constataram haver marcada heterogeneidade entre as empresas, em que pese o uso de pacotes tecnológicos semelhantes e a fruticultura, hoje, ser a atividade predominante. Portanto, este trabalho visa analisar a eficiência técnica das empresas agrícolas do pólo utilizando o modelo de função Translog para obter uma fronteira eficiente. A análise dos resultados mostra que o modelo apresenta escores de eficiência significantes. A análise comparativa entre os principais perímetros revela que a maior parte das firmas eficientes estão localizadas nos perímetros de Nilo Coelho e Maniçoba ou são externas aos perímetros.

\footnotetext{
* Mestre em Economia, Pós-Graduação em Economia, PIMES / Universidade Federal de Pernambuco (UFPE). Bolsista CAPES, cursando doutorado em Economia na Université-Pantheon-Sorbonne-Paris I, em Paris, França, desde 2002. e-mail: embarros2002@yahoo.fr ** Ph.D. em Economia Agrícola, University of Georgia. Professor do Departamento de Economia / PIMES, UFPE. Bolsista CNPq. Endereço: Praça Professor Fleming, 50 / Apto. 1.201 Recife, PE 52050-180. e-mail: ecio@yahoo.com

*** Ph.D. em Economia, University of California. Professor Titular do Departamento de Economia / PIMES, UFPE. Bolsista CNPq. e-mail: ysampaio@elogica.com.br
} 
Palavras-chave: Eficiência, irrigação, rentabilidade, translog.

Classificação JEL: Q12, Q15, Q16.

Abstract: The irrigation programs started in the late 60's were chiefly responsible for the impressive development occurred in the Petrolina/Juazeiro areas. Along with irrigation, came some agricultural enterprises that had been previously dedicated to other economic activities such as extensive cattle production. The main hypothesis of this study is that modern technology used in irrigation, combined with input usage, allow for a production level near or at the production frontier. Preliminary studies concluded that there is heterogeneity among enterprises, where similar technological packages are used and fruit production is the dominant production. Therefore, this study analyzes technical efficiency of agricultural enterprises in the studied area using a Translog function model to attain an efficient frontier. Results show that the model has significant efficiency scores. The comparative analysis among main irrigation areas shows that most of the efficient firms are located in the government delimited irrigation areas of Nilo Coelho and Maniçoba or outside delimited irrigation areas.

Keywords: Efficiency, irrigation, profitability, Translog

JEL Classification: Q12, Q15, Q16

\section{Introdução}

O pólo Petrolina/Juazeiro vem experimentando nas duas últimas décadas um crescente dinamismo (SAMPAIO e SAMPAIO, 2002). Isto se deve, em grande parte, à implantação de perímetros de irrigação, muitos deles iniciados nas décadas de 1960 e 1970. A produção, antes da implantação, limitava-se à pequena agricultura tradicional de sequeiro. A iniciativa do Setor Público viabilizou a utilização intensiva da terra em áreas de baixa densidade demográfica (típicas do semi-árido), e propiciou o estabelecimento de empreendimentos agrícolas e agroindustriais. Como conseqüência, isto gerou impactos diretos e indiretos sobre o emprego e a 
renda de grande magnitude, refreando também o êxodo rural (OLIVEIRA et al., 1991; SOUZA, 1995; e, VERGOLINO e VERGOLINO, 1987).

Dado esse quadro, o pólo se tornou atrativo aos investimentos privados, ao lado das áreas de colonos, nos perímetros irrigados. Empresas do Centro-Sul do país e do exterior vieram investir em fruticultura, atraídas pela possibilidade de produção durante o ano todo e a viabilidade de introdução de culturas de maior valor unitário e de base técnica mais elevada. Atualmente, o pólo está voltado para três atividades econômicas: fruticultura e olericultura irrigada, com potencial para diversificação de frutas exportáveis em larga escala; pecuária (ovina e caprina); e, agroindústria processadora de frutas e produtos agropecuários (BANCO DO NORDESTE, 2001).

Dentre as principais vantagens competitivas da região, pode-se destacar: disponibilidade de terra e água de boa qualidade; mão-de-obra abundante; infra-estrutura de irrigação implantada e em expansão; proximidade dos mercados europeu e norte-americano; e, ciclo produtivo mais precoce, com altos níveis de produtividade.

Os sentidos desta nova realidade estão fortemente direcionados a uma produção eficiente, eficaz e competitiva, capaz de introduzir-se no mercado externo, com elevado padrão de qualidade. As agroindústrias têm tido um papel fundamental nesse quadro, pois têm criado produtos derivados dessas novas culturas, agregando mais renda ao processo, além de gerar efeitos a montante e a jusante, através do encadeamento de setores direta e indiretamente ligados ao fornecimento de matériasprimas e máquinas, comercialização e distribuição.

Estudos recentes, no entanto, têm contido indicações de que há marcada heterogeneidade entre as empresas da região (SAMPAIO e SAMPAIO, 2002). Mas nenhum estudo foi realizado em relação à eficiência das empresas, em contraste com os colonos, para os quais há análises recentes de eficiência (SILVA e SAMPAIO, 2002). Estudos de eficiência, ainda que exploratórios, são importantes por constatar efetivamente a presença de heterogeneidades e propiciar condições para estudos mais aprofundados das suas causas e das políticas potenciais voltadas para atenuação das disparidades encontradas.

Portanto, este trabalho tem três objetivos principais. O primeiro é estimar a eficiência técnica das empresas agrícolas situadas no pólo Petrolina/Ju- 
azeiro através da agregação das culturas produzidas por cada uma dessas unidades produtoras. O segundo é comparar os escores de eficiência, obtidos através do método de fronteira de produção estocástica Translog. E, por último, verificar os resultados obtidos quanto ao rank de eficiência.

\section{Metodologia}

Os dados utilizados neste trabalho são provenientes da pesquisa "Investimentos Públicos e Privados em Agricultura Irrigada e Seus Determinantes sobre o Emprego e a Renda” (Fundação de Apoio ao Desenvolvimento da Universidade Federal de Pernambuco - FADE, 1999), a qual abrangeu observações de colonos, empresas agrícolas e empresas agro-industriais da região do Vale do São Francisco.

Cinqüenta e duas empresas foram pesquisadas, distribuídas nos perímetros de irrigação e fora destes. Dessas, somente 32 foram analisadas, sendo eliminadas as que apresentaram informações incompletas. A amostra inclui empresas localizadas nos perímetros Nilo Coelho (8), Maniçoba (7), Curaçá (2), e Bebedouro (2) e fora dos perímetros (13). Estes dados são cross-section, e referem-se apenas ao ano agrícola de 1998.

Os níveis de eficiência são obtidos utilizando o modelo paramétrico de estimação de fronteira estocástica, com distribuição half normal, através do Método de Máxima Verossimilhança. A função de produção Translog é utilizada como suposição básica quanto à função de produção.

Outro ponto a salientar é que a estimação da fronteira de produção é feita com a agregação de todos os produtos, usando como variável dependente o valor da produção das culturas irrigadas. As variáveis exógenas foram: área irrigada, que engloba a área cultivada com culturas irrigadas; insumos, representados pelos gastos com sementes, adubos, defensivos, herbicidas, água e energia; capital, que corresponde à soma dos custos com reparos de benfeitorias e equipamentos; e, mão-de-obra, que corresponde ao trabalho familiar e contratado, medido em homens/ano.

A escolha do valor da produção como variável dependente reside no fato de que esta variável é a que melhor representa o ganho (ou receita) de cada firma, podendo ser até considerada uma proxy para a variável quantidade produzida. 
Embora existam alguns desconfortos relativos ao uso de variáveis absolutas, pois poderiam mascarar o efeito preço e gerar distorções quanto aos ganhos de eficiência devido à variação da cesta entre as diferentes empresas, este trabalho leva também em conta que as empresas agrícolas sempre trabalham com cestas variadas de produtos e uma análise de eficiência a nível de produtores supõe uma agregação de produtos, já que, na maioria dos casos, os produtores não produzem uma única cultura.

\subsection{As Funções Fronteiras e a Análise de Eficiência}

A grande variedade de funções de produção e de custo conhecidas atualmente na literatura econômica nos fornece a possibilidade de obter diversos resultados sobre o comportamento de um dado conjunto de firmas, limitadas por uma determinada tecnologia. Além do mais, elas são bastante úteis no sentido de verificar se estão minimizando seus respectivos custos obtendo, assim, um nível de produção situado na fronteira de produção.

No entanto, a origem da análise de eficiência tem como ponto de partida o trabalho publicado por Farrel (1957) onde o autor utilizou algumas técnicas de programação linear para encontrar a eficiência econômica. Seu trabalho foi bastante simples e fez uso de uma isoquanta-unidade eficaz para estabelecer uma combinação de insumos e tecnologia que gerassem uma fronteira máxima de produção. Nenhuma forma funcional foi estabelecida inicialmente.

A eficiência econômica foi decomposta então em dois tipos: eficiência técnica e eficiência alocativa. No modelo de Farrel (1957), a eficiência técnica é definida como a habilidade que uma determinada firma tem de maximizar o nível de produção dados o conjunto de insumos e a tecnologia disponível. A eficiência alocativa, por outro lado, é definida como a medida do sucesso desta mesma firma na escolha de proporções ótimas de insumos, onde a taxa marginal de produção, para cada par de insumos, é igual à taxa de seus respectivos preços.

A partir deste trabalho inicial, vários tipos de modelos de estimação de funções fronteiras foram estabelecidos e novas contribuições foram adicionadas à análise ao longo das décadas de 1960 até 1980. Atualmen- 
te, os estudos sobre eficiência se concentram basicamente sobre duas principais abordagens: a paramétrica e a não paramétrica.

A abordagem paramétrica tem como principal característica a imposição de uma forma funcional à tecnologia. Ela se desenvolveu a partir dos trabalhos de Afriat (1972); Aignier e Chu (1972); Aigner, Lovell e Schmidt (1977); e, Meeusen e Van Den Broecker (1977). Eles foram os primeiros a supor uma forma funcional para explicar os níveis de eficiência das firmas e dividiram a abordagem paramétrica em dois grupos: os modelos com fronteiras deterministas e os modelos com fronteiras estocásticas.

A diferença básica entre este dois tipos de fronteiras reside na suposição com relação ao termo de erro. O modelo de fronteiras deterministas supõe que toda ineficiência é devido aos fenômenos que estão sobre o controle das firmas. O termo de erro possui uma distribuição unilateral não simétrica. Aignier e Chu (1972); e, Afriat (1972) foram os grandes defensores deste modelo. O modelo de fronteira estocástica, por outro lado, supõe dois tipos de erros: um erro unilateral, com as mesmas características pertencentes às fronteiras deterministas; e, um erro simétrico, com variação aleatória, que captura os efeitos ligados aos eventos fora de controle da firma e próprios das relações empíricas. O termo de erro no modelo de fronteira estocástica é considerado composto porque ele representa a união destes dois tipos de erros (unilateral e simétrico).

Geralmente, o termo de erro simétrico possui uma distribuição normal com média igual a zero e variância dada por $\sigma^{2}$. Aigner, Lovell e Schmidt (1977); e, Meeusen e van Den Broecker (1977) foram os primeiros a desenvolver uma função fronteira supondo um termo de erro composto.

Os modelos de fronteiras paramétricas utilizaram, na sua fase inicial, formas funcionais neoclássicas como as funções Leontief e Cobb-Douglas. Na década de 1980, com o surgimento das formas funcionais flexíveis, como a Translog, criou-se um novo campo de estudo, devido ao fato principal que estas novas formas funcionais não verificam algumas propriedades básicas das primeiras formas funcionais como, por exemplo, a elasticidade de substituição unitária e a concavidade ou a quase-concavidade das funções fronteiras em alguns dos seus pontos.

No entanto, devido ao fato da presença de elasticidade de substituição variável entre os insumos no quadro das formas funcionais flexíveis, observou-se que os níveis de eficiência obtidos com tais formas funcionais foram 
superiores aos obtidos com as formas funcionais neoclássicas. Isto tornou este grupo de funções atrativo e bastante utilizado nas estimações de funções fronteiras com resultados onde as estatísticas de testes não eram muito significativas nas estimações feitas com as formas funcionais neoclássicas. Huang e Bagi (1984); Kalirajan (1984); Kalirajan e Flinn (1983); Kalirajan (1990); e, Squires e Tabor (1991) são exemplos de trabalhos que estimaram os níveis de eficiência através da forma funcional flexível Translog.

A abordagem não paramétrica representa somente uma outra forma de conceber os níveis de eficiência. Ela foi desenvolvida diretamente da análise inicial de Farrel e sua característica mais marcante está na ausência de hipóteses que estabeleçam uma relação na qual os insumos são transformados em produtos, ou seja, na ausência de uma função de produção. Ela tem como regra básica a comparação de observações de “firmas práticas”, com auxílio de programação linear, para estabelecer uma fronteira com uma tecnologia de "melhor prática”, criada a partir de todas as combinações convexas de firmas disponíveis. As firmas situadas abaixo desta fronteira seriam consideradas ineficazes. De forma geral, as únicas restrições impostas à abordagem não paramétrica são que a tecnologia deve ser convexa (perfeita divisibilidade) e ter livre disponibilidade de insumos e produtos.

\subsubsection{Modelo de Fronteira Paramétrica Estocástica Translog}

A abordagem paramétrica de fronteira estocástica foi proposta inicialmente por Aignier e Chu (1972); e, Meeusen e Van Den Broecker (1977). Esta abordagem consistiu basicamente numa tentativa de superar as limitações das fronteiras determinísticas, que não permitiam a presença de erros aleatórios, considerando todos os resíduos como ineficiência técnica das firmas, controlados pelas mesmas.

Há muitos excelentes estudos sobre a evolução da estimação de fronteiras de eficiência. Entre esses, citam-se Silva (1999); Jondrow et al. (1982); Tulkens (1993); Banker (1996); e, Souza, Alves e Ávila (1997), tratando tanto de fronteiras paramétricas como não-paramétricas. Esses modelos têm sido bastante aplicados à agricultura brasileira (entre outros podemos citar CONCEIÇÃO e ARAUJO, 2000; SILVA, 1999; e, SILVA e SAMPAIO, 2002). De acordo com a visão determinística de modelo de 
fronteira, a tecnologia é representada por uma função dada por:

$$
y_{i}=f\left(x_{k} ; \beta_{k}\right)+u_{i}
$$

Onde $y_{i}$ é o vetor de produto, $x_{i k}$ representa o vetor de insumos, e $\boldsymbol{\beta}_{k}$ são os parâmetros a serem estimados e $\boldsymbol{u}_{i}$ representa o erro unilateral, captador da ineficiência técnica, de inteiro controle das firmas, sem a presença de erros aleatórios.

Já no modelo de fronteiras estocásticas, a estimação das fronteiras utiliza tecnologias que admitem um termo do erro dividido em duas partes: uma que mede a eficiência técnica, passível de controle pelas firmas; e, outra que captura erros aleatórios, fora do controle das firmas, tais como chuvas, estiagens, entre outros. A função de produção, nesse caso, é representada por uma função clássica Cobb-Douglas, dada por:

$$
\begin{aligned}
& y_{i}=f\left(x_{i k} ; \beta_{k}\right)+\varepsilon_{i} \\
& \varepsilon_{i}=v_{i}+u_{i} .
\end{aligned}
$$

Onde $\boldsymbol{y}_{i}$ é n nroduto da firma, $\boldsymbol{\beta}_{k}$ representa os parâmetros a serem estimados, $x_{i k}$ é o vetor de insumos. O termo $\boldsymbol{\varepsilon}_{i}$ representa o erro composto que está dividido em dois componentes: um, dado por $\mathbf{V}_{i}$, no qual frequentemente assume a forma de uma distribuição normal, capturando os erros aleatórios fora do controle da firma; e, outro, dado por $\boldsymbol{u}_{\boldsymbol{i}}$, com distribuição assimétrica, que mede a eficiência técnica através da diferença entre o produto observado e o produto na fronteira. O termo de erro assimétrico pode assumir, dependendo do tipo de estimação a ser feita e da qualidade dos dados, várias hipóteses quanto à lei estatística como, por exemplo, a half-normal, a exponencial, a gamma, dentre outras.

No caso de estimações através de funções de produção, este termo é não positivo $\left(\boldsymbol{u}_{\boldsymbol{i}} \leq \boldsymbol{0}\right)$ e revela que o produto de cada firma deve estar localizado sobre a fronteira ou abaixo dela. Se a análise utiliza funções custo, este termo será não negativo. Qualquer desvio é devido a fatores que estão dentro do controle das firmas como esforços do produtor, dos trabalhadores, entre outros. 
Conforme citado acima, embora existam vários tipos de hipóteses quanto à análise da distribuição deste termo de erro, como por exemplo, as distribuições exponencial e gamma, a maioria dos estudos de funções fronteiras (GREENE, 1980; GREENE, 1990; GREENE, 2000) sugerem a utilização de uma distribuição normal ou de uma quase-normal para este termo de erro. Portanto, baseando-se nestas hipóteses, este trabalho supõe uma distribuição quase-normal para o erro unilateral.

Os métodos clássicos de estimação para este tipo de fronteira são os de Máxima Verossimilhança e de Mínimos Quadrados Corrigidos (Corrected Ordinary Least Square, COLS). No entanto, o método de Mínimos Quadrados Corrigidos nem sempre funciona em algumas amostras, quando não é possível calcular o desvio padrão da distribuição half normal, $\boldsymbol{\sigma}_{u}$; e, o desvio padrão da distribuição white noise, $\boldsymbol{\sigma}_{v}$. Logo, será utilizado o método de Máxima Verossimilhança por ser mais abrangente na análise.

A função distribuição do erro composto é dada por:

$$
f\left(\varepsilon_{i}\right)=\sqrt{2} / \sigma_{u} \sqrt{\pi}\left[1-F\left(\varepsilon_{i} \lambda \sigma^{-1}\right)\right] \exp \left(-\varepsilon_{i}^{2} / 2 \sigma^{2}\right)
$$

Onde $\boldsymbol{\sigma}^{2}=\sigma_{u}{ }^{2}+\sigma_{v}{ }^{2}, \lambda=\sigma_{u} / \sigma_{v}$ e $F\left(^{*}\right)$ é a função distribuição acumulada da normal padrão. A função log likelihood será, então:

$\ln L\left(y / \beta, \lambda, \sigma^{2}\right)=N \ln \frac{\sqrt{2}}{\sqrt{\pi}}+N \ln \sigma^{-1}+\sum_{i=1}^{N} \ln \left[1-F\left(\varepsilon_{i} \gamma \sigma^{-1}\right)\right]-\frac{1}{2 \sigma^{2}} \sum_{i=1}^{N} \varepsilon_{i}^{2}$

Utilizando o método proposto por Jondrow et al. (1982), onde se considera o valor esperado de $\boldsymbol{u}_{\boldsymbol{i}}$ condicional a $\boldsymbol{\varepsilon}_{\boldsymbol{i}}$, em que $\boldsymbol{v}_{i}$ tem distribuição normal e $\boldsymbol{u}_{i}$ tem distribuição half normal, será possível estimar os índices de eficiência de cada empresa agrícola. Este valor esperado tem a forma:

$$
E\left(u_{i} / \varepsilon_{i}\right)=\mu_{0}+\sigma_{*} \frac{f\left(-\mu_{*} / \sigma_{*}\right)}{1-F\left(-\mu_{*} / \sigma_{*}\right)}
$$

Onde $f$ e $F$ são a função densidade e a função distribuição acu- 
mulada da normal, respectivamente,

$$
\frac{-\mu_{*}}{\sigma_{*}}=\varepsilon_{i} \lambda \sigma, \sigma^{2}=\sigma_{u}{ }^{2}+\sigma_{v}{ }^{2}, \sigma{ }^{2}=\sigma_{u}{ }^{2} \sigma_{v}{ }^{2} / \sigma^{2} \text { e } \lambda=\sigma_{u} / \sigma_{\nu}
$$

Uma vez obtidos os valores da distribuição de $\boldsymbol{u}_{\boldsymbol{i}}$, basta tomar a exponencial, $\exp \left(-E\left(u_{i} / \varepsilon_{i}\right)\right.$, e estimar a eficiência técnica de cada empresa agrícola, que estará situada no intervalo [0,1], com 0 representando completa ineficiência e 1 plena eficiência.

A forma funcional Translog representa um desdobramento da função Cobb-Douglas e é utilizado neste estudo por possivelmente apresentar uma maior flexibilidade das variáveis observadas ${ }^{1}$. O modelo de fronteira estocástica utilizando a função Translog estimado é definido por:

$\ln V P=\alpha+\beta_{1} \ln (A I)+\beta_{2} \ln (I N S)+\beta_{3} \ln (K)+\beta_{4} \ln (M O)+\beta_{5}\left(1 / 2 \ln ^{2} A I\right)+$ $\beta_{6}\left(1 / 2 \ln ^{2} I N S\right)+\beta_{7}\left(1 / 2 \ln ^{2} K\right)+\beta_{8}\left(1 / 2 \ln ^{2} M O\right)+\beta_{9} \ln A I \ln I N S+\beta_{10} \ln A I \ln K$ $+\beta_{11} \ln A I \ln M O+\beta_{12} \ln I N S \ln K+\beta_{13} \ln I N S \ln M O+\beta_{14} \ln K \ln M O+\varepsilon_{i}$.

Onde VP representa o valor da produção, $A I$ representa a área irrigada, INS os gastos com insumos, $K$ os gastos com capital e $M O$ traduz a quantidade de mão-de-obra utilizada. O valor da produção e os gastos com insumos foram medidos em reais; a área irrigada em hectares; o capital representou o somatório dos custos com reparos de benfeitorias e equipamentos; e, a mão-de-obra foi medida em homens/dia. Como já mencionado, o termo $\varepsilon_{i}$ é composto por dois componentes, dados por $\boldsymbol{\varepsilon}_{i}=\boldsymbol{v}_{i}+\boldsymbol{u}_{i}$, onde $\boldsymbol{v}_{i}$ representa erros aleatórios, fora do controle da firma como estiagens, chuvas, entre outros, dados por uma distribuição normal; e, $\boldsymbol{u}_{\boldsymbol{i}}$ representa o erro unilateral que mede a eficiência técnica através da diferença entre o produto observado e o produto na fronteira, com distribuição half normal.

A principal diferença entre as funções de produção Cobb-Douglas e Translog é que a primeira trabalha com retornos de escala restritos aos mesmos valores para todas as firmas da amostra e elasticidade de

\footnotetext{
${ }^{1} \mathrm{O}$ modelo Translog foi testado em comparação ao modelo Cobb-Douglas e se adaptou melhor aos dados aqui utilizados, segundo Barros, Costa e Sampaio (2004).
} 
substituição unitária. A Translog relaxa estas suposições dando maior flexibilidade ao modelo. A idéia por trás desse pressuposto é que, devido à maior flexibilidade, os escores de eficiência sejam mais elevados que os da Cobb-Douglas. Outro ponto a salientar é que o modelo estocástico utiliza níveis relativos de eficiência, pois é raro encontrar firmas com eficiência perfeita, igual a um. Isto se deve, em grande parte, ao fato de assumirmos um formato para a função de produção. A função Translog, por exemplo, tende a quantificar escores perto do valor unitário, talvez devido à sua maior flexibilidade.

\section{Resultados}

Estatísticas descritivas das 32 empresas que são analisadas neste trabalho são apresentadas na Tabela 1. Embora exista grande disparidade entre os valores mínimo e máximo, a maior parte das firmas utiliza os fatores disponíveis em patamares bastante equilibrados, necessários à garantia de valores de produção eficientes. Em média, as 32 firmas utilizaram 73,19 hectares de área irrigada com uma mão-de-obra de 65,82 homens/ano.

Tabela 1. Estatísticas descritivas das variáveis usadas no modelo Translog

\begin{tabular}{llrrrr}
\hline \multicolumn{1}{c}{ Variável } & \multicolumn{1}{c}{ Unidade } & \multicolumn{1}{c}{ Média } & Desvio-Padrão & \multicolumn{1}{c}{ Mínimo } & \multicolumn{1}{c}{ Máximo } \\
\hline Valor da Produção & Real (R\$) & $787.358,62$ & $859.904,18$ & $4.167,00$ & $3.060 .708,00$ \\
Área Irrigada & Hectare & 73,19 & 94,69 & 2,00 & 444,00 \\
Gastos com Insumos & Real (R\$) & $24.147,95$ & $22.218,93$ & 66,60 & $80.940,00$ \\
Gastos com Capital & Real (R\$) & $52.618,38$ & $36.953,05$ & $9.056,36$ & $161.239,40$ \\
Mão-de-obra & Homens/dia & 66 & 106,38 & 0,92 & 610,00 \\
\hline
\end{tabular}

Os resultados quanto à estimação da fronteira estocástica Translog estão explícitos na Tabela 2. As variáveis significantes foram insumos, mão-deobra, o quadrado da área irrigada, o quadrado dos gastos com insumos, o quadrado do capital, a interação entre área irrigada e capital, a interação entre insumo e capital, a interação entre insumo e mão-de-obra, e, a interação entre capital e mão-de-obra. Contudo, a variável insumo e todas as suas possíveis interações apresentaram sinal contrário ao esperado. Talvez devido à má utilização dos insumos disponíveis, como o excesso de gastos 
com adubos, defensivos, ou até com água e energia. A interação entre capital e área irrigada apresentou sinal negativo. Isto pode ser explicado pelo fato de que um aumento em hectares na área utilizada para irrigação acarreta maiores despesas com maquinarias e novos equipamentos, de modo a manter a produção com o mesmo padrão de qualidade existente antes da expansão da área irrigada. As demais variáveis apresentraram sinal igual ao esperado. Estes resultados mostram que os efeitos das interações existentes entre os diversos fatores permitem uma melhor visão do que possivelmente está afetando a eficiência das firmas.

Tabela 2. Resultados das estimativas da função de fronteira estocástica Translog

\begin{tabular}{|c|c|}
\hline Variável & Coeficientes \\
\hline Intercepto & $20,7924 *$ \\
\hline Área irrigada & 1,1614 \\
\hline Insumos & $-4,0375^{*}$ \\
\hline Capital & 0,3490 \\
\hline Mão-de-obra & $3,0808^{*}$ \\
\hline Área Irrigada ${ }^{2}$ & $0,1125^{*}$ \\
\hline Insumos $^{2}$ & $-0,0854^{*}$ \\
\hline Capital $^{2}$ & $-0,2393 *$ \\
\hline Mão-de-obra $a^{2}$ & 0,0623 \\
\hline Área Irrigada x Insumos & 0,5915 \\
\hline Área Irrigada $\times$ Capital & $-0,6372^{*}$ \\
\hline Área Irrigada x Mão-de-obra & $-0,1469$ \\
\hline Insumos $x$ Capital & $0,5847^{*}$ \\
\hline Insumos x Mão-de-obra & $-0,9311 *$ \\
\hline Capital x Mão-de-obra & $0,6144^{*}$ \\
\hline$\sigma$ & 0,0122 \\
\hline$\sigma_{u}^{2}$ & $4,5398 \mathrm{E}-07$ \\
\hline$\mu \underset{v}{2}$ & 0,6602 \\
\hline Log da função likelihood & $-17,6005$ \\
\hline
\end{tabular}

* Significante ao nível de $5 \%$.

Os escores de eficiência encontrados utilizando o modelo Translog foram relevantes. Comparando as cinco empresas mais eficientes com as cinco empresas menos eficientes (Tabela 3) pode-se ver o contraste que envolve cada grupo. Ambos produzem uma variedade de culturas que possuem alto valor unitário e que são exportadas com facilidade, como, 
por exemplo, uva e manga. A diferença é que as mais eficientes souberam utilizar melhor os recursos disponíveis, como a área irrigada, a quantidade de trabalhadores, insumos, entre outros, de modo a agregar um maior valor à produção, enquanto que as menos eficientes talvez tenham utilizado de forma um pouco desordenada os recursos que estavam em seu poder, como a contratação de mão-de-obra excessiva, o não aproveitamento eficiente da área irrigada, os gastos elevados, entre outros.

A comparação entre as cinco mais eficientes com as cinco menos eficientes deste modelo mostra que a característica básica das cinco mais eficientes é a utilização racional dos recursos disponíveis de modo a agregar um maior valor à produção. O valor médio da produção dessas empresas foi de R \$ 1.093.557,00, com utilização de uma área irrigada média de 98 hectares e de 43 homens/ano. As cinco menos eficientes mantiveram baixos níveis de valor agregado de produção, perfazendo o total médio de R $\$$ 270.281,00 utilizando 37 hectares de área irrigada e 45 homens/ano. Essas empresas devem apresentar alguma deficiência no uso dos demais fatores. Talvez existam problemas na interação de fatores, que podem, dependendo do tamanho do impacto dessa interação, ocasionar uma queda no valor da produção e, consequentemente, fazer o nível de eficiência decrescer. Ao se observar estes valores comparativamente com os apresentados na Tabela 1, vê-se que a área irrigada média do modelo Translog, para as cinco mais eficientes, foi maior que a área irrigada média das empresas como um todo e menor em termos de mão-de-obra em relação ao nível de mão-de-obra médio total. Isto revela outras possibilidades de alocação de recursos de modo a garantir melhores níveis de eficiência.

Tabela 3. Dados referentes às firmas mais eficientes $v$ s. menos eficientes

\begin{tabular}{ccccccc}
\hline Rank & Escore & $\begin{array}{c}\text { Valor da } \\
\text { Produção } \\
\text { (reais) }\end{array}$ & $\begin{array}{c}\text { Área Irrigada } \\
\text { (ha) }\end{array}$ & $\begin{array}{c}\text { Valor da Produção / } \\
\text { Área Irrigada } \\
\text { (reais/ha) }\end{array}$ & $\begin{array}{c}\text { Mão-de-obra } \\
\text { (homens/ano) }\end{array}$ & $\begin{array}{c}\text { Mão-de-obra } \\
\text { / Área Irigada } \\
\text { (homens/ano/ha) }\end{array}$ \\
$1^{\text {a }}$ & 0,9997 & 470.750 & 62 & 7593 & 67 & 1,08 \\
$2^{\text {a }}$ & 0,9991 & 929.620 & 16 & 58101 & 66 & 4,13 \\
$3^{\text {a }}$ & 0,9988 & 246.600 & 77 & 3203 & 14 & 0,18 \\
$4^{\text {a }}$ & 0,9838 & 3.064 .875 & 322 & 9518 & 66 & 0,20 \\
$5^{\text {a }}$ & 0,9765 & 755.938 & 13 & 58149 & 4 & 0,30 \\
\hline $28^{\mathrm{a}}$ & 0,2502 & 929.620 & 55,2 & 16841 & 66 & 1,19 \\
$29^{\mathrm{a}}$ & 0,2037 & 141.136 & 21,0 & 6721 & 18 & 0,84 \\
$30^{\mathrm{a}}$ & 0,1993 & 175.000 & 40,0 & 4375 & 65 & 1,63 \\
$31^{\mathrm{a}}$ & 0,1450 & 74.500 & 42,0 & 1774 & 7 & 0,16 \\
$32^{\mathrm{a}}$ & 0,1241 & 31.150 & 25,2 & 1236 & 67 & 2,68 \\
\hline
\end{tabular}


Outro ponto a ser levado em conta é que os escores de eficiência no modelo Translog chegaram perto da unidade, ou seja, essas firmas mostraram-se bastante eficientes, enquanto que as ineficientes tiveram seus escores perto de zero. Isto ocorreu devido à flexibilidade desse tipo de função, que não impõe restrições à elasticidade de substituição unitária como no caso de uma função Cobb-Douglas, por exemplo. A Tabela 4 ilustra os escores de eficiência distribuídos em classes. A distribuição apresenta que $34,37 \%$ das firmas estão situadas no intervalo entre 0,2 e 0,6, mas o maior peso está entre o intervalo 0,6 a 1 , com $56,26 \%$ das firmas situadas nesta região. Há nove firmas perto do nível ótimo de eficiência, representando $28,13 \%$ do total.

Tabela 4. Escores de eficiência para o modelo paramétrico estocástico Translog

\begin{tabular}{lcc}
\hline Classes de Eficiência & Número de firmas & $\%$ \\
\hline 0 a 0,2 & 3 & 9,37 \\
0,2 a 0,4 & 4 & 12,50 \\
0,4 a 0,6 & 7 & 21,87 \\
0,6 a 0,8 & 9 & 28,13 \\
0,8 a 1 & 9 & 28,13 \\
Total & 32 & 100 \\
\hline
\end{tabular}

Ao analisarmos o nível de eficiência segundo os perímetros de irrigação e as firmas externas aos perímetros, encontramos resultados significantes. Para efeito de análise, consideram-se como eficientes as empresas que obtiveram escores no intervalo de 0,6 a 1 . As firmas com níveis de eficiência entre 0,6 e 1 estão situadas nos perímetros Bebedouro, Curaçá, Maniçoba, Nilo Coelho e externas aos perímetros. A Tabela 5 mostra este quadro. O número de firmas eficientes do modelo perfaz um total de dezoito.

Tabela 5. Distribuição das firmas eficientes entre os perímetros de irrigação

\begin{tabular}{lccc}
\hline Perímetros & Número de firmas & Firmas eficientes & \% (por perímetro) \\
\hline Bebedouro & 2 & 1 & 50,00 \\
Curaçá & 2 & 2 & 100,00 \\
Maniçoba & 7 & 5 & 71,43 \\
Nilo Coelho & 8 & 3 & 37,50 \\
Externas & 13 & 7 & 53,85 \\
\hline
\end{tabular}


Outra comparação interessante está na Tabela 6. Ela apresenta a localização de cada uma das cinco firmas mais eficientes, ou seja, o perímetro em que cada uma delas está instalada. Nos resultados obtidos, 3 das 5 mais eficientes são externas aos perímetros, uma está localizada no perímetro Maniçoba e outra no perímetro Nilo Coelho, sendo que as duas mais eficientes estão fora dos perímetros. Isto vem demonstrar que o fator localização pode exercer grande influência na quantificação do escore de eficiência.

Tabela 6. Comparação da localização das firmas mais eficientes

\begin{tabular}{ccc}
\hline Rank & Escore & Perímetro \\
\hline $1^{\text {a }}$ & 0,9997 & Externas \\
$2^{\text {a }}$ & 0,9991 & Externas \\
$3^{\text {a }}$ & 0,9988 & Nilo Coelho \\
$4^{\text {a }}$ & 0,9838 & Externas \\
$5^{\text {a }}$ & 0,9765 & Maniçoba \\
\hline
\end{tabular}

\section{Conclusões}

A maioria das variáveis apresentou sinal igual ao esperado, com a exceção da variável insumo e todas as suas possíveis interações apresentaram sinal contrário ao esperado. A interação entre capital e área irrigada apresentou sinal negativo. Isto pode ser explicado pelo fato de que um aumento na área utilizada para irrigação acarreta maiores despesas com maquinarias e novos equipamentos.

Os escores de eficiência permitem uma comparação entre os valores médios das variáveis valor da produção, área irrigada e mão-de-obra para as 32 empresas analisadas e os valores médios dessas variáveis para o modelo Translog entre as firmas eficientes e ineficientes, revelando que as firmas ineficientes possuem baixo valor de produção, com excesso de área irrigada e pouca mão-de-obra. Recomenda-se análise das tecnologias adotadas, bem como da organização das empresas, que permita aprofundar as razões da ineficiência.

Os escores de eficiência chegaram perto da unidade, ou seja, algumas firmas mostram-se bastante eficientes enquanto que as ineficientes tiveram seus escores perto de zero. Isto se deve à flexibilidade desse tipo de função, que não impõe restrições à elasticidade de substituição unitária. 
A análise comparativa entre os principais perímetros revelou ainda que a maior parte das firmas eficientes estão localizadas nos perímetros de Nilo Coelho e Maniçoba ou são externas aos perímetros. Esta constatação mostra que a experiência de implantação dos perímetros tanto foi bem sucedida em atrair empresas eficientes para os perímetros como para induzir a instalação de empresas igualmente eficientes fora dos mesmos.

As empresas localizadas fora dos perímetros são mais eficientes devido, provavelmente, ao fato de que os perímetros possuem uma parte considerável de suas áreas compostas por pequenos empresários. Além disso, a cultura do empreendorismo pode não ser tão representativa nestes perímetros, dado que estes perímetros, e suas empresas localizadas dentro dos mesmos, há muito tempo recebem grande apoio do governo federal através de assistência técnica, cobrança diferenciada pelo uso da água, sistemas de irrigação previamente instalados, entre outros, gerando ineficiências de produção e de alocação de recursos. Pode-se, assim, concluir que as empresas localizadas fora dos perímetros tenham que apresentar um comportamento mais eficiente, pois não contam com o mesmo apoio daquelas localizadas em perímetros.

\section{Referências bibliográficas}

Afriat, S. N. "Efficiency Estimates of Production Functions”. International Economic Review. 13: 568-598. 1972.

Aigner, D. J. e S. F. Chu. “On Estimating The Industry Production Function”. American Economic Review. 13(3): 568-598. 1972.

Aigner, D. J., C. K. Lovell e P. Schmidt. "Formulation and Estimation of Stochastic Frontier Production Function Models”. Journal of Econometrics. 6: 21-37. 1977.

Banco do Nordeste do Brasil - BNB. http://www.bancodonordeste.gov. br. Acesso em 2001.

Banker, R. D. "Hypothesis Tests Using Data Envelopment Analysis”. The Journal of Productivity Analysis. 7: 138-158. 1996.

Barros, E. S., E. F. Costa e Y. Sampaio. "Análise de Eficiência Através da Estimação de Fronteiras Paramétricas Cobb-Douglas e Translog: O Caso 
das Empresas Agrícolas do Pólo Petrolina/Juazeiro.” Revista Econômica do Nordeste. 35(1):7-19. 2004.

Conceição, J. C. P. R. e P. F. C. de Araujo. "Fronteira de Produção Estocástica e Eficiência Técnica na Agricultura”. Revista de Economia e Sociologia Rural. 38(1):45-64. 2000.

Fundação de Apoio ao Desenvolvimento da Universidade Federal de Pernambuco - FADE. Investimentos Públicos e Privados em Agricultura Irrigada e seus Impactos sobre o Emprego e a Renda nos Pólos de Petrolina/Juazeiro e Norte de Minas Gerais. Recife, Março, 1999.

Farrell, M. J. "The Measurement of Economic Efficiency". Journal of the Royal Statistical Society. 120, Series A, Pt. III: 252-281. 1957.

Greene, W. H. (2000), Econometric Analysis. Fourth Edition, New York University.

Greene, W. H. “A Gamma Distributed Stochastic Frontier Model”. Journal of Econometrics. 46: 141-163. 1990.

Greene, W. H. "Maximum Likelihood Estimation of Econometric Frontier Functions”. Journal of Econometrics. 13: 27-56. 1980.

Huang, C. J. e F. S. Bagi. "Technical Efficiency in a Traditional Indian Agriculture”. Southern Economic Journal. 51: 233-238. 1984.

Jondrow, J., C. A. Lovell, S. Materov e P. Schmidt. "On Estimation of Technical Inefficiency in the Stochastic Frontier Production Function Model”. Journal of Econometrics. 19: 233-238. 1982.

Kalirajan, K. "Farm-Specific Technical Efficiencies and Development Policies”. Journal of Economics Studies. 11: 3-13. 1984.

Kalirajan, K e J. C. Flinn. "The Measurement of Farm-Specific Technical Efficiency”. Pakistan Journal of Applied Economics. 2: 167-180. 1983.

Kalirajan, K. “On measuring Economic Efficiency”. Journal of Applied Econometrics. 5: 75-85. 1990.

Meeusen, W. e J. Van Den Broeck. "Efficiency Estimation from Cobb-Douglas Production Function with Composed Error”. International Economic Review. 18(2): 435-444. 1977. 
Oliveira, A. C., H. R. Souza, J. R. Vergolino, O. A. Galvão e A. Melo. Impactos Econômicos da Irrigação Sobre o Pólo Petrolina/Juazeiro. Ed. Universitária, Recife. 1991.

Sampaio, Y. e E. Sampaio. Ensaios Sobre a Economia da Agricultura Irrigada. Ed. Universitária, Recife. 356p. 2002.

Silva, J. M. A Eficiência dos Colonos na Agricultura Irrigada no Vale do São Francisco. Tese de Doutorado. PIMES/UFPE. 1999.

Silva, J. M. e Y. Sampaio. "A Eficiência Técnica dos Colonos na Agricultura Irrigada no Vale do São do Francisco". Economia Aplicada. 6(2): 265-285. 2002.

Souza, G. S., E. Alves e A. F. D. Ávila. "Produtividade e Eficiência Relativa de Produção em Sistemas de Produção de Pesquisa Agropecuária”. Revista Brasileira de Economia. 51(3): 281-307. 1997.

Souza, H. R. "Agricultura Irrigada no Semi-Árido Nordestino”. In: Gomes, G. M., H. R. Souza e A. R. Magalhães. Desenvolvimento Sustentável no Nordeste. IPEA, Brasília, 1995.

Squires, D. e S. Tabor. “Technical Efficiency and Future Production Gains in Indonesian Agriculture”. The Developing Economies. 258-270. 1991.

Tulkens, H. "An FDH Efficiency Analysis: Some Methodological Issues and Applications to Retail Banking, Courts, in Urban Transit”. The Journal of Productivity Analysis. 4: 183-210. 1993.

Vergolino, T. e J. R. Vergolino. Relações de Trabalho e Condições de Vida dos Trabalhadores na Agricultura Irrigada do Submédio do São Francisco. Relatório de Pesquisa, PIMES/UFPE. 1987. 